\title{
Research on Ultrasonic Vibration Milling in Optical Glass Materials
}

\author{
Jie Zhao ${ }^{1, a}$, Yanyan Zhao ${ }^{2, b}$, Guofu Gao ${ }^{3, c}$ \\ ${ }^{1}$ Henan college of industry \& information technology, China \\ ${ }^{2}$ Henan Polytechnic University, China \\ ${ }^{3}$ Henan Polytechnic University, China

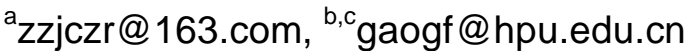

Keywords: Ultrasonic vibration milling; Optical glass; Milling force; Multi-factor orthogonal experiment method.

Abstract. With the rapid development of aerospace, optics, photonics and other fields, the optical glass is more and more widely used. In ultrasonic vibration milling process, the milling force can directly affect the stability of machining process, the processing quality of components and the pass rate of products. In this paper, the experiment on ultrasonic vibration and ordinary milling of optical glass studied the influence of milling speed, feed per tooth, milling depth and other factors for the milling force. The experimental results showed that, compared with the ordinary milling process, the ultrasonic milling can effectively reduce the milling force and improve the processing stability in the same processing conditions.

\section{Introduction}

Optical glass is more and more widely used in aerospace, optics, photonics and other fields. Because of its high brittleness and low fracture toughness, it is too difficult to obtain high surface quality by using ordinary processing methods. Moreover, the qualified rate of products is too low and the processing cost is too high. Ultrasonic vibration milling by pulse cutting as its main characteristic is a new kind of processing technology, which not only can reduce the cutting force, effect of cutting heat and the machined surface roughness, but also can greatly improve the machining accuracy and extend the tool life. For difficult-to-machining materials, especially for precision machining of the hard and brittle materials, Ultrasonic vibration milling reflects more benefits. Noma Kazuki proposed that the axial ultrasonic vibration-assisted machining with workpiece bending has the potential for achieving high-precision and high-efficiency machining for chemically strengthened glass [1].Zhang Chenglong established the Mathematical model for cutting force in rotary ultrasonic face milling of brittle materials in his paper [2]. Bulla B. studied influence on the ductile behaviour of binderless tungsten carbide applying ultrasonic assisted diamond turning [3]. In the process of ultrasonic vibration milling, the milling force determines the machining quality and precision of the workpiece [4,5].In this paper, multi-factor orthogonal experiment method with different experimental parameters is used to research the influence rules of different processing factors on the milling force so that the optimal parameters combination for optical glass ultrasonic vibration milling is obtained.

\section{Model of Ultrasonic Vibration Milling Force}

In the same cutting conditions, ultrasonic vibration milling is the result of both ordinary cutting and ultrasonic vibration, so the average milling force acting on the workpiece (the same effecting on the tools) is the superposition of the ordinary milling force $S$ and the average impact force during the machining. In the process of ultrasonic vibration milling, the average milling force of the cutting tool $[6,7]$ is:

$$
F=S+\bar{F}=S+\frac{2 \pi f^{2} A M\left(\cos \left(2 \pi f t_{e}\right)-\cos \left(2 \pi f t_{s}\right)\right)}{\left(t_{e}-t_{s}\right) f+1}
$$


In above equation, $\cos \left(2 \pi f t_{e}\right)-\cos \left(2 \pi f t_{s}\right)<0$ and $\left(t_{e}-t_{s}\right) f+1=\left(t_{e}+T\right)-t_{s}>0$ so, $F=S+\bar{F}<S$. It shows that the average milling force $F$ produced in the ultrasonic vibration milling is smaller than $S$ that in the ordinary milling. Therefore, using ultrasonic vibration machining can effectively reduce the cutting force.

\section{Experimental Conditions and Method}

\section{Experimental Conditions of Milling}

In this experiment, the model ZK1 of optical glass is selected as the workpiece materials whose performance is shown in table 1 and machine tool, cutting tool are in table 2.

Tab.1 Experiment materials

\begin{tabular}{c|c|c|c}
\hline Elastic Modulus (E) & $\begin{array}{c}\text { Poisson Coefficient } \\
(\mu)\end{array}$ & Proportion & $\begin{array}{c}\text { Shear Modulus } \\
(\mathrm{G})\end{array}$ \\
$5.487 \mathrm{GPa}$ & 0.265 & $3.08 \mathrm{~g} / \mathrm{cm} 2$ & $3.33 \mathrm{GPa}$ \\
\hline
\end{tabular}

Tab.2 Experiment device

\begin{tabular}{ll}
\hline Machine Tool & Vertical Machining Center (Model: XH714B) \\
Cutting Tool & PCD Milling Cutter Diameter: $\Phi 12 \mathrm{~mm}$, Helix \\
& Angle $0^{\circ}$, Teeth number 4 \\
\hline
\end{tabular}

\section{Experimental Method}

The ultrasonic vibration milling system used to the optical glass materials in the experiment is shown in Figure 1 . This system mainly consists of an ultrasonic generator, a three-dimensional dynamometer, a multichannel charge amplifier by the type of 5070, a computer and so on. The dynamometer used in the test is Kistler 9257B which is made in Switzerland by Kistler Company.

In this experiment, ultrasonic vibration milling and ordinary milling are used to the workpiece to research the effect of the three main factors, milling speed (spindle speed $n$ ) Vc, feed rate (feed per tooth fz) f and cutting depth ap on milling force. Meanwhile, down milling is selected as the milling mode. Cutting fluid is kerosene and frequency of ultrasonic vibration is $36.85 \mathrm{KHz}$. Multi-factor orthogonal experiment method is used to do the test of milling force.

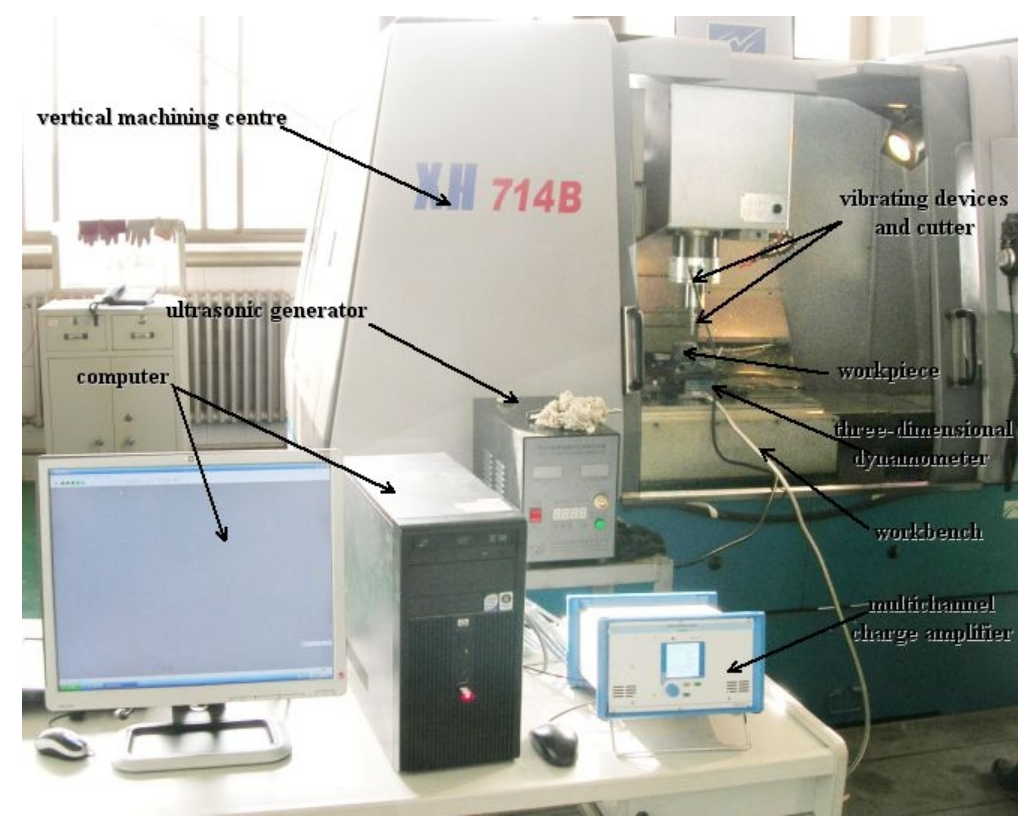

Fig.1 Ultrasonic vibration milling system 


\section{Analysis of the Influence of Various Factors on Milling Force}

Influence of Milling Speed on Milling Force. The relation curve between the milling force in three directions(Fx, Fy and Fz) and the milling speed in ultrasonic vibration and ordinary milling is showed in Figure 2. The Figure 2 shows that the milling force Fx, Fy and Fz are all increasing along with the milling speed in the two milling ways. As the milling speed from $226 \mathrm{~m} / \mathrm{min}$ to $264 \mathrm{~m} / \mathrm{min}$, the milling force increases sharply. Moreover, the milling vibration is obvious too when the milling speed is increasing. The milling force enlarges, because the strain rate of the workpiece is increasing along with the milling speed and the cutting volume increases in the unit time. It is showed that in Figure 2 the milling force $\mathrm{Fx}$, Fy and $\mathrm{Fz}$ in ultrasonic vibration milling are all $12 \sim 75 \mathrm{~N}$ lower than the ordinary milling. This explains the ultrasonic vibration milling can effectively reduce the milling force in processing in some certain conditions, especially the $\mathrm{Y}$ and $\mathrm{Z}$ direction, and then improve the stability of milling process.

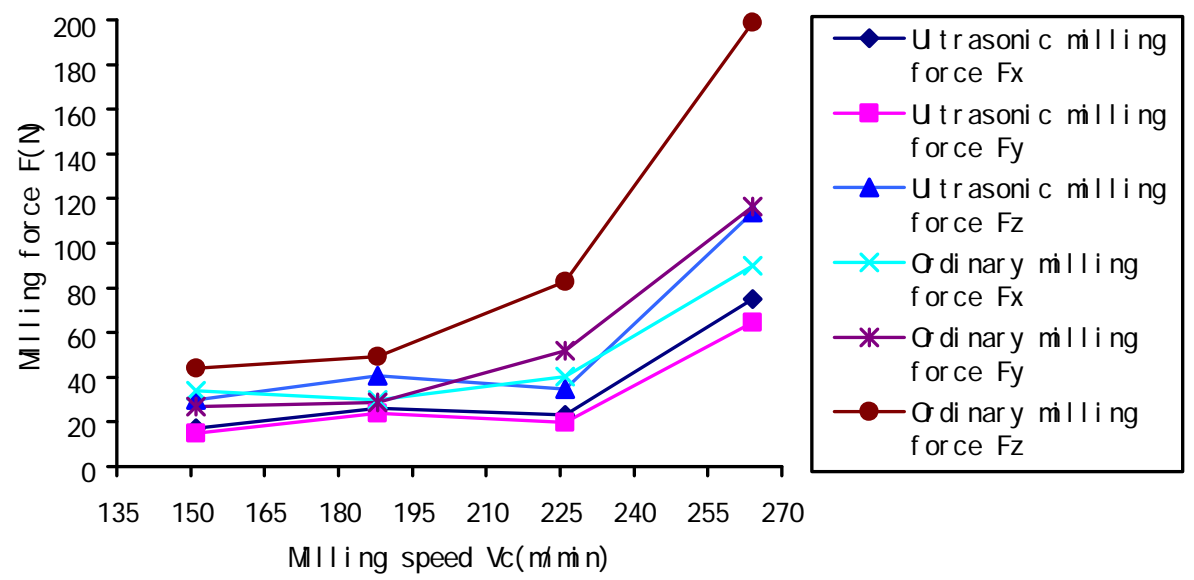

Fig.2 The influence of milling speed on milling force in each direction

Influence of Feed Rate per Tooth on Milling Force. In the two machining ways of ultrasonic vibration and ordinary milling, the relation curve between the milling force in three directions (Fx, Fy and Fz) and the feed per tooth is showed in Figure 3. It is can be seen from Figure 3 that in the two milling ways, the milling force in each direction Fx, Fy and Fz generally increases with the increase of the feed rate. The change of milling force in three directions is very obvious in ordinary milling process, but in ultrasonic vibration milling is not. This shows that the milling force produced in ultrasonic vibration milling is much smaller than that in ordinary milling. Besides, in ultrasonic vibration milling, the increase of the feed rate has produced little effect on the milling force in $\mathrm{Y}$ and $\mathrm{Z}$ directions but much in X. It illustrates that the ultrasonic vibration milling can significantly reduce the milling force in $\mathrm{Y}$ and $\mathrm{Z}$ directions and make the change of it more uniform in $\mathrm{X}$ when compared with ordinary milling.
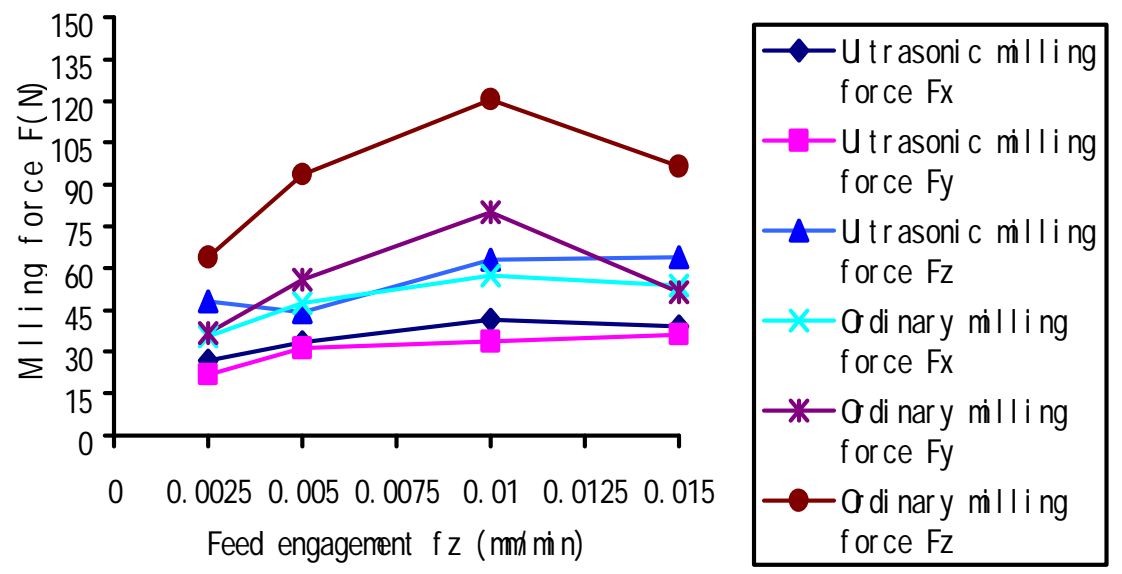

Fig.3 The influence of feeding on each direction milling force 
Influence of Milling Depth on Milling Force. The relation curve between the milling force in three directions(Fx, Fy and Fz) and the milling depth in ultrasonic vibration and ordinary milling is showed in Figure 4. The Figure 4 shows that with the increase of the milling depth, the milling force is increasing too in both two milling methods. When the milling depth is increasing, the removal of the materials in unit time and the energy consumption in milling process are increasing. At the same time, due to the increasing of the milling depth, the cutting edge and contact area between the cutting tool and workpiece are all increased, so the friction is increased which causes the enlargement of the milling force. However, compared with ordinary milling, the milling force in three directions(Fx, Fy and Fz) are all much lower in ultrasonic vibration milling. It explains that the ultrasonic vibration milling can reduce the milling force and improve the stability of the processing.

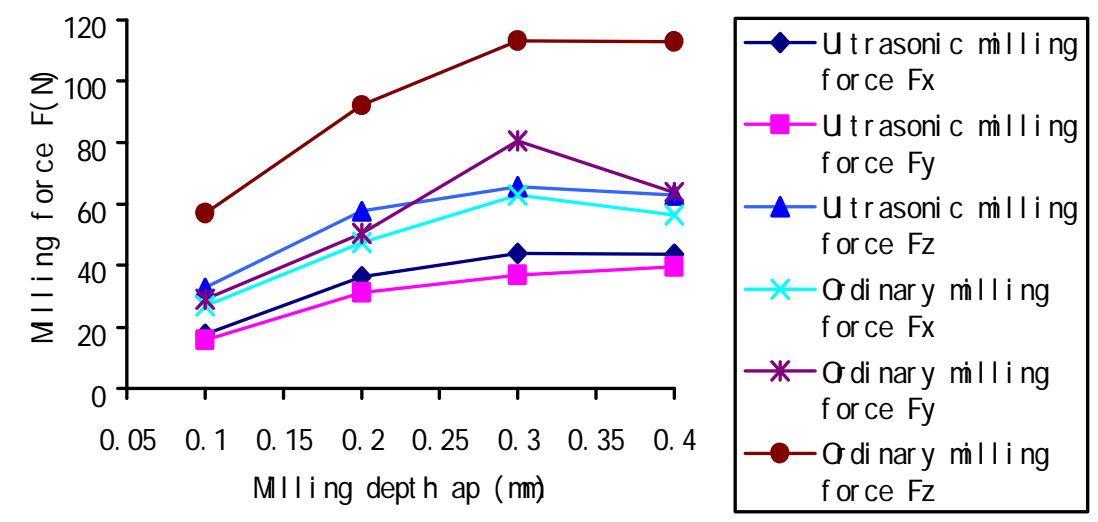

Fig. 4 Influence of Milling Depth on Milling Force

\section{Conclusions}

The experiment in this paper shows that the milling force is increasing with the increase of the milling speed, feed per tooth and milling depth, but the increase of the milling force is much small and increasing evenly in ultrasonic vibration milling. Meanwhile, the milling force can be significantly reduced in ultrasonic vibration milling compared with ordinary milling in the same cutting conditions. Therefore, the ultrasonic vibration milling can effectively reduce the milling force and improve the stability of the machining process, then improve the surface quality of the workpiece.

\section{References}

[1] Noma, Kazuki. Ultrasonic vibration-assisted machining of chemically strengthened glass with workpiece bending , Journal of Advanced Mechanical Design, Systems and Manufacturing, v 9, n 2, 2015

[2] Zhang, Chenglong. Mathematical model for cutting force in rotary ultrasonic face milling of brittle materials , International Journal of Advanced Manufacturing Technology, v 69, n 1-4, p 161-170, 2013

[3] Bulla, B. Influence on the ductile behaviour of binderless tungsten carbide applying ultrasonic assisted diamond turning, Proceedings of the 13th International Conference of the European Society for Precision Engineering and Nanotechnology, EUSPEN 2013, v 2, p 229-232, 2013

[4] Zhou Hongwei, Gu Meilin et al. Research on milling force in ultrasonic vibration micro milling of transparent quartz glass [J]. Tool Engineering, 2014, 48(5): 32-35

[5] Zhang Qiaoe, Xiang Daohui. Research on cutting force and tool wear of high volume SiCp/Al composites with ultrasonic vibration high-speed milling [J]. Editorial Board of Journal of HPU (Nature Science), 2013, 32(2): 209-212 
[6] Qu Weide, Tang Hengling. Handbook of mechanical vibration [M]. Beijing: China Machine Press, 2000

[7] Ken-ichi Ishikawa, Hitoshi Suwabe, Tetsuhiro Nishid and Michio Uneda, A study on combined vibration drilling by ultrasonic and low-frequency vibrations for hard and brittle materials [J]. Precision Engineering, 1998, (12): 196-205 